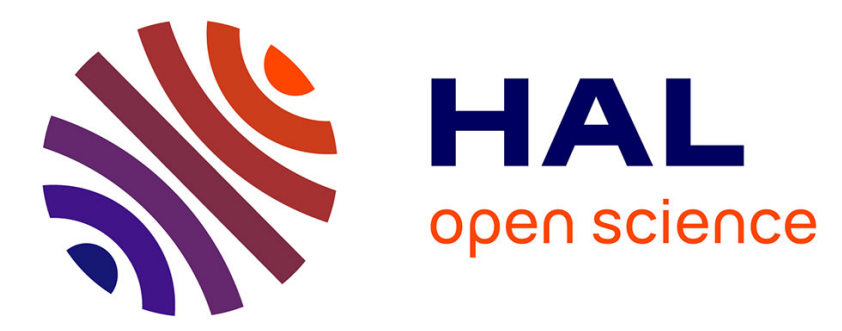

\title{
Outputs analysis and dual immersion method for chaotic systems
}

\author{
Kokou A.A. Langueh, Octaviana Datcu, Jean-Pierre Barbot, Gang Zheng, \\ Krishna Busawon
}

\section{- To cite this version:}

Kokou A.A. Langueh, Octaviana Datcu, Jean-Pierre Barbot, Gang Zheng, Krishna Busawon. Outputs analysis and dual immersion method for chaotic systems. International Conference on Computing, Communication and Security, ICCCS 2015, PROF. SUBRAMANIAM GANESAN, Dec 2015, Pointe Aux Piments, Mauritius. hal-01240249

\section{HAL Id: hal-01240249 \\ https://hal.science/hal-01240249}

Submitted on 8 Dec 2015

HAL is a multi-disciplinary open access archive for the deposit and dissemination of scientific research documents, whether they are published or not. The documents may come from teaching and research institutions in France or abroad, or from public or private research centers.
L'archive ouverte pluridisciplinaire HAL, est destinée au dépôt et à la diffusion de documents scientifiques de niveau recherche, publiés ou non, émanant des établissements d'enseignement et de recherche français ou étrangers, des laboratoires publics ou privés. 


\title{
Outputs analysis and dual immersion method for chaotic systems.
}

\author{
K.A.A. Langueh, O. Datcu, J-P Barbot, G. Zheng and K. Busawon* \\ December 5, 2015
}

\begin{abstract}
When secure data transmission is implemented through chaotic systems, the choice of the output is a preliminary problem. In this paper, the quality of the transmitted information is analysed with respect to the observability concept, for each potential output. Moreover, in order to overcome observability loss, a dual immersion technique is proposed.The use of high order sliding mode observer on a well known Lorenz system, allows to highlight the well founded of the proposed analysis and method.
\end{abstract}

Keywords: Chaotic systems, Observability, Singularity, Immersion, Secure data transmission.

\section{Introduction}

Since the works of Pecora and Caroll [19], it is well known that two chaotic systems can be synchronized. Following this fact, many authors have proposed secure data transmission schemes (see for example $[5,1,17]$ ) based on the synchronization of chaotic systems. Nevertheless, the output choice

*K.A.A Langueh is with QUARTZ Laboratory EA 7393, ENSEA, 6 Avenue du Ponceau, 95014 Cergy-Pontoise, O. Datcu is with Politehnica University of Bucharest, Faculty of Electronics, Telecommunications, and Information Technology and QUARTZ Laboratory, IPGP, ENSEA, 6 Avenue du Ponceau, 95014 Cergy-Pontoise, J-P. Barbot is with QUARTZ Laboratory EA 7393, ENSEA, 6 Avenue du Ponceau, 95014 Cergy-Pontoise, EPI Non-A, INRIA. G. Zheng is with EPI Non-A INRIA, Lille, Nord-Europe and K. Busawon is with Northumbria University, Newcastle, UK. 
with respect to the synchronization, at the best of our knowledge, is less analyzed (see for example [13, 9]). In [18] Marels and Nijmeijer draw the link between unidirectional synchronization and observation. This allows to use the control system theory to analyze the synchronization of chaotic systems. Nevertheless, as chaotic systems are always nonlinear systems, linear control system theory can't be directly applied to such systems. This is due to the fact, for example, basic properties as stability, controllability and observability are generally local for nonlinear systems. It is the reason why in [11] local observability concepts and criteria are introduced. These lead to determine an observability singularity set [9]. This set is only constituted of 'forbidden' states (i.e. state which are unobservable). In this paper, starting from the results introduced in [11] and other recent works [13, 9] an analysis of the "best" output for secure data transmission is realized on the basic Lorenz circuit. It is important to mention that the observability analysis with respect to the output choice is only a preliminary step in the design of data secure transmission scheme. After that, many other problems occur, such as the choice of the ciphering method, the input choice, the method for retrieving information (see for example $[6,12]$ ),... Moreover, when an output is chosen, it may exist an observability singularity set, which leads to lose information in some part of the state space. Hereafter it is proposed a solution, based on the immersion technique, to overcome this problem under very weak conditions. It is important to mention that, since the work of $[22,21]$, the immersion technique was extensively used in the observer design context. Nevertheless, this is usually used to recover the linearity property by diffeomorphism and output injection [3, 4, 24, 23], but generally with only a local diffeomorphism. In the majority of the mentioned papers, immersion was accomplished by adding a dynamic by means of output integration [3]. The stability of such extra dynamics can be problematic and an elegant solution is proposed in [23]. Nevertheless the problem of observability singularity was not tackled. A dual immersion technique using only extra differentiations is proposed in this paper. This method is close to the one proposed in [2], in another context. Moreover, for stability arguments it is chosen, in this paper, to impose exponentially stable dynamics instead of constant dynamics. The proposed approach is feasible thanks to the finite time differentiators as for example the one proposed in [7] (but for this method, the delay appears due to the data acquisition frame) or High Order Sliding Mode (HOSM) [15, 8].

The paper is organized as follows: in the next section some observability concepts, symbolic observability index and observability singularity defini- 
tions are recalled and the problem statement is explained in the framework of Lorenz system. In section III, the dual immersion method is presented. After that, some recalls on HOSM differentiator are given in section IV. In section V, simulation examples, with respect to Lorenz system, are given. The paper ends with some conclusions and perspectives.

\section{Some recalls and problem statement}

Consider the following autonomous system:

$$
\begin{aligned}
& \dot{x}=f(x) \\
& y=h(x)
\end{aligned}
$$

where $x \in \mathbb{R}^{n}$ is the state, and $y \in \mathbb{R}$ is the output. $f$ and $h$ are supposed to be $C^{\infty}$ vector fields. Rougthly speaking, in [11] in order to determine if or not the system (1) is observable (weakly locally observable) Hermann and Krener have introduced the following condition:

Proposition 1 The system (1) is weakly locally observable if

$$
\operatorname{Rank}\{d \mathcal{O}(\infty)\}=n
$$

where

$$
d \mathcal{O}(\infty)_{\left.\right|_{x_{0}}}=\left(\begin{array}{c}
d h \\
\vdots \\
d L_{f}^{n-1} h \\
\vdots \\
d L_{f}^{n+k} h \\
\vdots
\end{array}\right)_{\left.\right|_{x_{0}}}
$$

In (3) $L_{f}^{i} h=\frac{\partial L_{f}^{i-1} h}{\partial x} f$ is the usual Lie derivative and $d L_{f}^{i} h=\left(\frac{\partial L_{f}^{i} h}{\partial x_{1}}, \frac{\partial L_{f}^{i} h}{\partial x_{2}}, \ldots, \frac{\partial L_{f}^{i} h}{\partial x_{n}}\right)$ is the associated 1 -form.

Remark 1 If the system (1) is linear, then the weakly locally observability implies the classical global observability. Moreover, due to the Cayley Hamilton theorem, it is sufficient to compute the $n$ first rows of $d \mathcal{O}(\infty)$ (denoted $d \mathcal{O}(n))$. 
Starting, from this linear argument and the fact that it is natural to design an observer of the same dimension that the original system in [20] the concept of regularly weakly locally observability was introduced. Roughly speaking, only the $n-1$ output derivatives are considered for recovering all the state. This leads to the following condition:

Proposition 2 The system (1) is regularly weakly locally observable if

$$
\operatorname{Rank}\{d \mathcal{O}(n)\}=n
$$

with

$$
d \mathcal{O}(n)_{\left.\right|_{x_{0}}}=\left(\begin{array}{c}
d h \\
\vdots \\
d L_{f}^{n-1} h
\end{array}\right)_{\left.\right|_{x_{0}}}
$$

Since $d \mathcal{O}(n)_{\left.\right|_{x_{0}}}$ is only of full rank $n$ around $x_{0}$, this implies that there might exist some $\bar{x} \in \mathbb{R}^{n}$ such that rank $d \mathcal{O}(n)_{\mid \bar{x}}<n$. Due to this fact, let us define the following observability singularity set:

$$
\mathcal{S}_{n}=\left\{x \in \mathbb{R}^{n}: \operatorname{rank}\left\{d \mathcal{O}(n)_{\left.\right|_{x}}\right\}<n\right\}
$$

In this case, for the purpose of removing the singularities in $\mathcal{O}(n)_{\left.\right|_{x}}$ defined in (6), it is necessary to increase the dimension of (5) by involving more derivatives of the output. This will be the purpose of the next section, but before, an example of the output choice is given hereafter.

Example 1 Let us consider the Lorenz system

$$
\begin{aligned}
& \dot{x}_{1}=\sigma\left(x_{2}-x_{1}\right) \\
& \dot{x}_{2}=r x_{1}-x_{2}-x_{1} x_{3} \\
& \dot{x}_{3}=-b x_{3}+x_{1} x_{2}
\end{aligned}
$$

Where $x=\left[x_{1}, x_{2}, x_{3}\right]^{T} \in \mathbb{R}^{3}$ is the state. Moreover, the parameter are chosen as follow $\sigma=10, r=28$ and $b=\frac{8}{3}$.

Case 1: The output $y=x_{1}$

For this output $d \mathcal{O}(3)_{\left.\right|_{x}}$ is equal to

$$
d \mathcal{O}(3)_{\left.\right|_{x}}=\left(\begin{array}{ccc}
1 & 0 & 0 \\
-\sigma & \sigma & 0 \\
\sigma\left(r+\sigma-x_{3}\right) & -\sigma(\sigma+1) & -\sigma x 1
\end{array}\right)_{\left.\right|_{x}}
$$




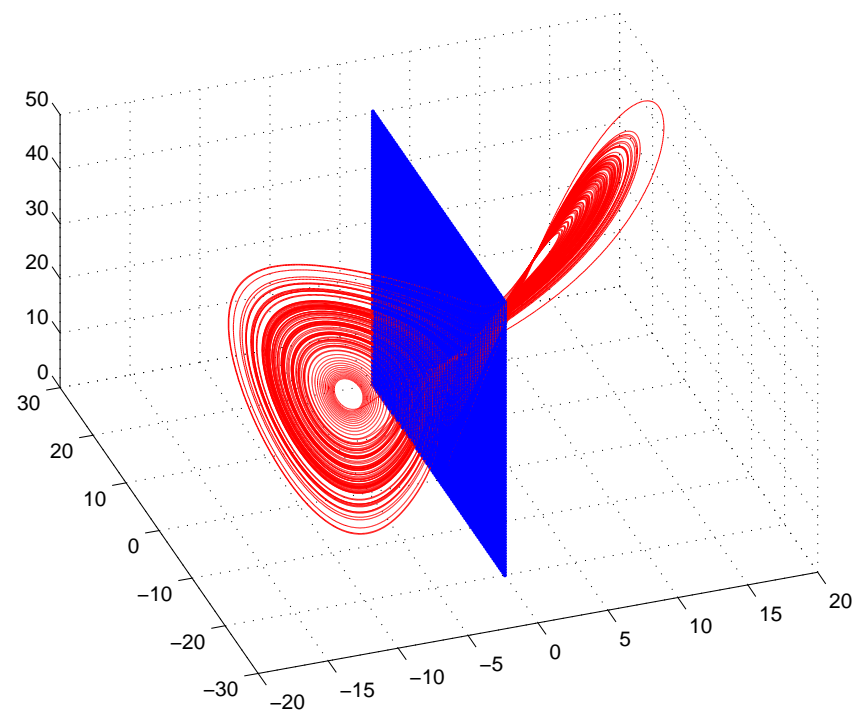

Figure 1: Lorenz attractor and the map $\mathcal{S}_{3}$

and then

$$
\mathcal{S}_{3}=\left\{x \in \mathbb{R}^{3}: x_{1}=0\right\}
$$

On the figure 1 it is shown that the set of observability singularity intersect the strange attractor. In fact the observability property is lost because only two derivatives of the output $y=x_{1}$ are considered. Nevertheless, if the third derivative is also considered $d \mathcal{O}(4)_{\mid x}$ becomes:

$$
d \mathcal{O}(4)_{\left.\right|_{x}}=\left(\begin{array}{ccc}
1 & 0 & 0 \\
-\sigma & \sigma & 0 \\
\sigma\left(r+\sigma-x_{3}\right) & -\sigma(\sigma+1) & -\sigma x_{1} \\
A & B & C
\end{array}\right)_{\left.\right|_{x}}
$$

where $A=-\sigma\left[\sigma^{2}+r \sigma(2 \sigma+1)+2 x_{1} x_{2}-(2 \sigma+b+1) x_{3}\right], B=\sigma\left[\sigma^{2}+\sigma(r+\right.$ $\left.1)+1-x_{1}^{2}-\sigma x_{3}\right]$ and $C=\sigma\left[(2 \sigma+b+1) x_{1}-\sigma x_{2}\right]$. Then, the observability singularity set is:

$$
\mathcal{S}_{4}=\left\{x \in \mathbb{R}^{3}: x_{1}=x_{2}=0\right\}
$$

this set does not intersect the strange attractor, see figure 2. Moreover, it is important to note that on $\mathcal{S}_{4}$, the system (7) is stable and consequently its behaviour is not chaotic. 


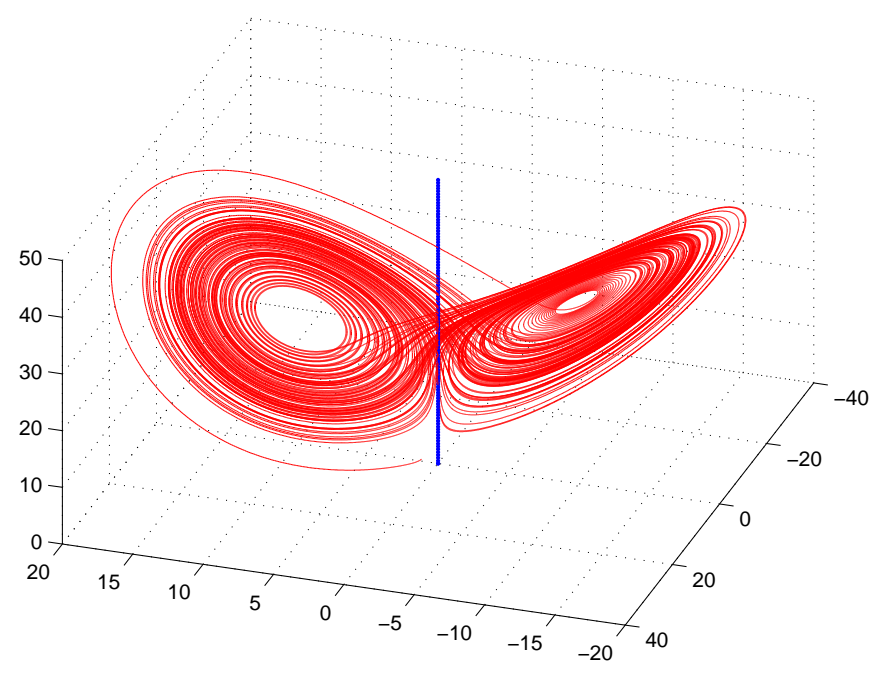

Figure 2: Laurenz attractor and the line $\mathcal{S}_{4}$

Case 2: The output $y=x_{2}$

In the same way as the case 1 , it is obtained

$$
\mathcal{S}_{3}=\left\{x \in R^{3}: 2 x_{2} x_{1}^{2}-b r x_{1}+r \sigma x_{2}-\sigma x_{2} x_{3}=0\right\}
$$

Case 3: The output $y=x_{3}$

Finally, for the third case, it is obtained

$$
\mathcal{S}_{3}=\left\{x \in R^{3}: 2 \sigma x_{2}^{2}-2 r x_{1}^{2}+2 * x_{1}^{2} x_{3}=0\right\}
$$

Comparing the set of observability singularities, for the sake of simplicity and space in the next only the firs case is considered.

The previous example, case 1 , has shown that, even if the $\mathcal{O}(3)$ contains singularities, i.e. its rank is not equal to 3 for some $x \in \mathbb{R}^{n}$, it is still possible to obtain a higher dimensional map: $\mathcal{O}(4)$ which will not contain singularity on the strange attractor. Thus it may be interesting to design an observer of greater dimension than 3 which is equivalent to use a state space representation greater than 3. More precisely, (9) was obtained from the following injective function:

$$
\mathcal{O}(4)=\left(y, \dot{y}, \ddot{y}, y^{(3)}\right)^{T}
$$


Remark 2 By extension $\mathcal{O}(n+k)$ is defined as follow:

$$
\mathcal{O}(n+k)=\left(y, \dot{y}, \ddot{y}, \ldots, y^{(n+k)}\right)^{T}
$$

Setting $z=\mathcal{O}(4)$, the extended dynamics becomes

$$
\dot{z}=\frac{\partial \mathcal{O}(4)}{\partial x} f(x)_{\mid x}=\left(\begin{array}{c}
z_{2} \\
z_{3} \\
z_{4} \\
L_{f}^{3} h(x)
\end{array}\right)
$$

for which a traditional high-gain observer [10] (or sliding mode observer [15]) can be easily designed to estimate $z$, noted as $\hat{z}$. Then, one can obtain the estimation of $x$, denoted $\hat{x}$ by:

$$
\hat{x}=\underset{\hat{x} \in \mathbb{R}^{n}}{\arg \min }\|\hat{z}-\phi(\hat{x})\|
$$

which can be seen as an optimization problem. For the above optimization framework, the essential point is that the Jacobian of $\mathcal{O}(4)$ is a matrix of 4 by 3 , thus non square, and this will cause difficulties when applying numerical methods to solve this optimization problem.

Motivated by this consideration, for a given $\mathcal{O}(n+k)$ defined in (11), hereafter, it is proposed to find a diffeomorphism $\phi$ by increasing the original state space. In the literature, this technique is called immersion and many authors use this immersion technique in order to obtain a specific normal form [23]. Most often, the immersion is realized by means of output integration. In this paper, it will be realized by a dual method which will be presented in the next section.

\section{Dual immersion technique}

\subsection{Preliminary results}

For a given $d \mathcal{O}(n)$ containing the singularities belonging to $\mathcal{S}_{n}$, this section will propose a constructive way to deduce an injective function $\mathcal{O}(n+k)$ defined in (11) by increasing the output derivative order. Then the following result is trivial. 
Lemma 1 Consider the observability singularity set $\mathcal{S}_{n}$ defined in (6), then the generated set $\mathcal{S}_{n+i}$ for $i \geq 1$ via differentiation is always included in $\mathcal{S}_{n+i-1}$, i.e.

$$
\mathcal{S}_{n+i} \subseteq \mathcal{S}_{n+i-1}
$$

Proof. For $i \geq 0$, the following equation

$$
\mathcal{O}(n+i)=\left[\begin{array}{c}
\mathcal{O}(n+i-1) \\
L_{f}^{n+i_{1}} h
\end{array}\right]
$$

is verified, hence

$$
\operatorname{rank}\{d \mathcal{O}(n+i)\} \geq \operatorname{rank}\{d \mathcal{O}(n+i-1)\}
$$

which implies that

$$
\mathcal{S}_{n+i} \subseteq \mathcal{S}_{n+i-1}
$$

Before presenting the dual immersion technique, the following assuption is reclame

Assumption 1 It is assumed that system (1) is globally observable for $x \in$ $\mathbb{R}^{n}$, or at least observable in the domain of interest $D$, i.e. there exists a least an integer $k \in \mathcal{Z}^{+}$such that

$$
\operatorname{rank}\{d \mathcal{O}(n+k)\}=n
$$

for all $x \in \mathbb{R}^{n}$, or at least for all $x \in D$, where $\mathcal{O}(n+k)$ is defined in (11).

With the above assumption, we can state the following result.

Lemma 2 For system (1), if Assumption 1 is satisfied, then there exists at least an integer $k \in \mathcal{Z}^{+}$such that the following inclusion is satisfied:

$$
\emptyset=\mathcal{S}_{n+k} \subseteq \cdots \subseteq \mathcal{S}_{n+1} \subseteq \mathcal{S}_{n}
$$

on $R^{n}$, or at least in D. Moreover, $z=\mathcal{O}(n+k)$ is such that rank $\{d \mathcal{O}(n+$ $k)\}=n$, on $R^{n}$, or at least in $D$.

Proof. The above result is a direct consequence of the previous lemma.

The above results show that one can reduce the observability singularity set by just increasing the number of the derivative of the output. Consequently, one can find at least an integer $k \in \mathcal{Z}^{+}$such that $z=\mathcal{O}(n+k)$ 
has a Jacobian of rank $n$ on $R^{n}$, or at least in $D$. However, its Jacobian is not square ( a matrix of $(n+k) \times n)$. In what follows, we will propose a constructive way to calculate a new diffeomorphism $\phi(x, \xi)$ from the deduced $\mathcal{O}(n+k)$ in Lemma 2 via the technique of dual immersion, whose Jacobian $d \phi(x)$ will be square and invertible, at least on $D$.

\subsection{Dual immersion approach}

Consider again system (1), and suppose that we have already found a least integer $k \in \mathcal{Z}^{+}$such that $z=\mathcal{O}(n+k)$ is a global injective function. Then let us present the following procedure to compute a global (or at least in $D$ ) diffeomorphism $\phi(x, \xi)$ (where $\xi \in \mathbb{R}^{k}$ will be defined hereafter).

Dual immersion algorithm

1. Initialization: Set $z_{1}=\phi_{1}(x)=y$;

2. Compute $z_{j}=\phi_{j}(x)=y^{(j-1)}$ until a singularity of observability appears in the row $d L_{f}^{(j-1)} h$;

3. Define $\phi_{j}=y^{(j-1)}+\xi_{1}$ where $\xi_{1}$ is an additional state, with the following dynamics:

$$
\dot{\xi}_{1}=-\epsilon_{1} \xi_{1}
$$

initialized at $\xi_{1}=0$.

4. - If $L_{f}^{(j+1)} h$ is not considered in (11), define $\phi_{i, j+1}=L_{f}^{(j)} h-\epsilon_{1} \xi_{1}$

- If $L_{f}^{(j+1)} h$ is considered in (11), define $\phi_{i, j+1}=L_{f}^{(j)} h-\epsilon_{1} \xi_{1}+\xi_{2}$, with

$$
\dot{\xi}_{2}=-\epsilon_{2} \xi_{2}+\dot{\epsilon}_{1} \xi_{1}-\epsilon_{1}^{2} \xi_{1}
$$

5. repeat the operation until the last line of (11) is reached. The resulting matrix $d\{\phi\}$ becomes a square matrix and if there exist $\epsilon_{j}$ such that the matrix is regular for all $x$ then the algorithm 3.2 converges.

This algorithm gives

Theorem 1 For system (1), if Assumption 1 is satisfied and Algorithm 3.2 converges then $\phi(x, \xi)$ with $\xi \in \mathbb{R}^{k}$ is a global, or at least on $D$, diffeomorphism. Moreover, the Jacobian of the generated $\phi(x, \xi)$ via the dual immersion satisfies: 


$$
\operatorname{rank} d \phi(x, \xi)=n+k
$$

for all $x \in \mathbb{R}^{n}$, or at least in $D$.

Proof. The proof follows from the algorithm, and assumption 1 is a necessary condition for the convergence of algorithm 3.2.

Roughly speaking, the dual immersion algorithm works as follow: considering that for the order $r=n+1, \mathcal{O}(r)$ generates an "acceptable" (see [9] for chaotic systems) observability singularity set $\mathcal{S}_{r}$ and the singularity problem arrives in the last line of $\mathcal{O}(n)$, it is possible to define a new change of coordinate $z=\phi(x, \xi)$ as follows: $z_{j}=y^{(j-1)}$ until $j=n-1$ (i.e. until such a problem of observability singularity appears in the row $\left.d L_{f}^{(n-1)} h\right)$. and for $j=n, z_{j}$ are assigned equal to $y^{(j-1)}+\xi_{1}$ where $\xi_{1}$ is an extra state, which verifies the following dynamics:

$$
\dot{\xi}_{1}=-\epsilon_{1} \xi_{1}
$$

initialized at $\xi_{1}=0$. Finaly for $j=n+1, z_{j}$ are assigned equal to $y^{(j)}+\dot{\xi}_{1}=$ $\dot{z}_{n}$.

Remark 3 From the stability and the initialization of the dynamics (14) it is not necessary to simulate this dynamic in the observer design because it is equivalent of adding zero at $y^{(j-1)}$. In [2] the proposed solution consists to adding a constant in order to be able to design a High gain observer in the original coordinates.

\section{Recalls on high-order sliding-mode}

In this paper, the proposed method is based on the so-called real-time exact robust HOSM differentiator $[15,16]$, which is recalled in the following.

Consider a signal $y(t) \in C^{k}$ (at least $k$ times differentiable), let us suppose $\left(y, \cdots, y^{(k)}\right)=\left(z_{1}, \cdots, z_{k+1}\right)$. The HOSM robust differentiator proposed in 
[15] takes the following form:

$$
\begin{aligned}
\dot{\hat{z}}_{1} & =-\lambda_{0} M^{\frac{1}{k}}\left|\hat{z}_{1}-y\right|^{\frac{k}{k+1}} \operatorname{sign}\left(\hat{z}_{1}-y\right)+\hat{z}_{2} \\
\dot{\hat{z}}_{2} & =-\lambda_{1} M^{\frac{1}{k-1}}\left|\hat{z}_{2}-v_{1}\right|^{\frac{k-1}{k}} \operatorname{sign}\left(\hat{z}_{2}-v_{1}\right)+\hat{z}_{3} \\
\vdots & \\
\dot{\hat{z}}_{k} & =-\lambda_{k-1} M^{\frac{1}{2}}\left|\hat{z}_{k}-v_{k-1}\right|^{\frac{1}{2}} \operatorname{sign}\left(\hat{z}_{k}-v_{k-1}\right)+\hat{z}_{k+1} \\
\dot{\hat{z}}_{k+1} & =-\lambda_{k} M \operatorname{sign}\left(\hat{z}_{k+1}-v_{k}\right)
\end{aligned}
$$

where $M$ is chosen to be bigger than the $k$-th derivative of $y(t), \lambda_{i}$ are positive design parameters, and the adjustment of those parameters is described in detail in [15]. Define the observation errors as: $e_{i}=z_{i}-\hat{z}_{i}$, thus the observation errors dynamics is given by:

$$
\begin{aligned}
e_{1} & =\hat{z}_{1}-y \\
e_{2} & =\dot{e}_{1}=\lambda_{0} M^{\frac{1}{k}}\left|e_{1}\right|^{\frac{k}{k+1}} \operatorname{sign}\left(e_{1}\right) \\
\vdots & \\
e_{k} & =\dot{e}_{k-1}=\lambda_{k-1} M^{\frac{1}{2}}\left|e_{k-1}\right|^{\frac{1}{2}} \operatorname{sign}\left(e_{k-1}\right) \\
e_{k+1} & =\dot{e}_{k}=\lambda_{k} M \operatorname{sign}\left(e_{k}\right)
\end{aligned}
$$

It has been proven in [14] that there exists $t_{0}$ such that $\forall t>t_{0}$ we have

$$
e_{i}=z_{i}-\hat{z}_{i}=0 \quad \text { for } \quad 1 \leq i \leq k+1
$$

In the next section, a Lorenz example case 1 will be simulated and commented.

\section{Lorenz example and immersion.}

Consider again the system (7) with $y=x_{1}$, with initial conditions $x_{1}(0) \neq 0$, $x_{2}(0) \neq 0$ and $x_{3}(0) \neq 0$. It was shown, in the second section that $\mathcal{S}_{4}=$ $\left\{x \in R^{3}: x_{1}=x_{2}=0\right\}$ and that this set of observability singularity is not on the strange attractor, thus as $D$ (the considered domain) is the strange attractor, then it is possible to use the dual immersion method.

Then $\mathcal{O}(n+k)$

$$
\phi(x)=[y, \dot{y}, \ddot{y}, \dddot{y}]^{T}
$$


has a regular Jacobian on $D$. So, the immersion is implemented as follows:

$$
z=\phi(x, \xi)=\left(\begin{array}{c}
y \\
\dot{y} \\
\ddot{y}+\xi \\
\dddot{y}+\dot{\xi}
\end{array}\right)
$$

with $\dot{\xi}=-\varepsilon \xi$ and $\xi(0)=0$ as initial condition. Then $d \phi(x, \xi)$ is a square matrix:

$$
d \phi(x, \xi)=\left(\begin{array}{cccc}
1 & 0 & 0 & 0 \\
-\sigma & \sigma & 0 & 0 \\
\sigma\left(r+\sigma-x_{3}\right) & -\sigma(\sigma+1) & -\sigma x_{1} & 1 \\
A & B & C & -\varepsilon
\end{array}\right)
$$

with $A=-\sigma\left[\sigma^{2}+r \sigma(2 \sigma+1)+2 x_{1} x_{2}-(2 \sigma+b+1) x_{3}\right], B=\sigma\left[\sigma^{2}+\sigma(r+\right.$ 1) $\left.+1-x_{1}^{2}-\sigma x_{3}\right]$ and $C=\sigma\left[(2 \sigma+b+1) x_{1}-\sigma x_{2}\right]$.

The $\varepsilon$ parameter is chosen such that $d \phi(x, \xi) \neq 0$ (i.e. $\varepsilon x_{1} \neq(2 \sigma+b+1) x_{1}-$ $\left.\sigma x_{2}\right)$ on $D$. Thus, as no derivative of $\varepsilon$ is considered in this exemple, this parameter is chosen as follows:

$\varepsilon$ is equal to $10^{4}$ if $\operatorname{det}\left\{\left.d \bar{\phi}(x, \varepsilon)\right|_{\varepsilon=10^{4}}\right\} \neq 0$ else $\varepsilon=10$, this ensuring that $\operatorname{det}\left\{d \bar{\phi}(x, \varepsilon)\right.$ is equal to zero only if $x_{1}=x_{2}=0$.

As the HOSM observer gives $\hat{z}=z$ in finite time, the estimated of $x$ is also obtain in finite time as follows:

$$
\begin{gathered}
x=\left.\phi^{-1}(z)\right|_{\varepsilon}= \\
{\left[\begin{array}{l}
z_{1} \\
\frac{\sigma z 1+z 2}{\sigma} \\
{\left[\begin{array}{l}
x_{3} \\
\xi
\end{array}\right]=\left[\begin{array}{cc}
-\sigma y & 1 \\
\sigma\left[(2 \sigma+b+1) y-\sigma x_{2}\right] & -\varepsilon
\end{array}\right]^{-1}\left[\begin{array}{l}
L_{1} \\
L_{2}
\end{array}\right]}
\end{array}\right]}
\end{gathered}
$$

with $L_{1}=\ddot{y}+(\sigma+1) \dot{y}-\sigma(r-1) y$ and $L_{2}=\dddot{y}-\left[\sigma^{2}+\sigma(r-1)+1\right] \dot{y}+$ $\left[\sigma^{2}(r-\sigma-1)+\sigma r\right] y+(\sigma y+\dot{y}) y^{2}$

The simulation was done with initial conditions chosen randomly in $[0,1]$ for the system and the observer, moreover on the HOSM observer the paramer $M$ is equal to $10^{6}$. In Fig. 3, the exact values of is obtained after $1 s$. 


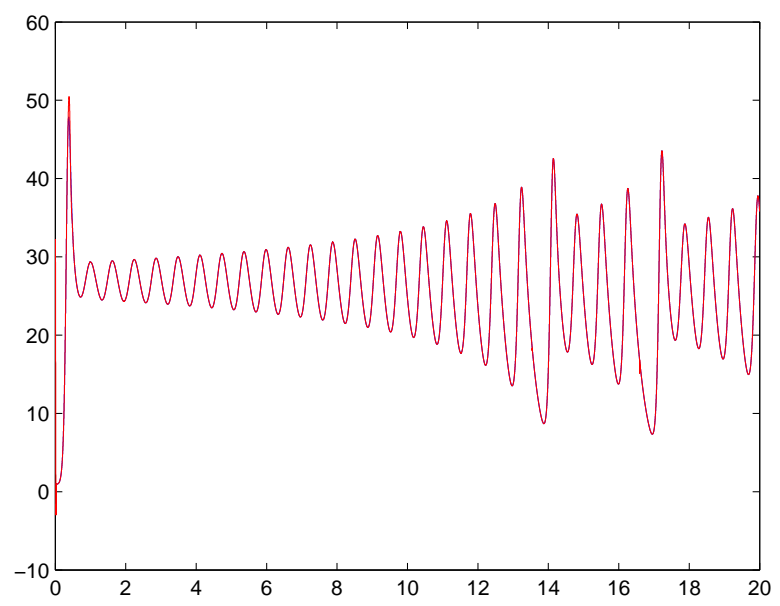

Figure 3: The stat $x_{3}$ and its estimate $\hat{x}_{3}$

\section{Conclusion}

In this paper an analysis of the observability, with respect to different outputs, was done. After that, a dual immersion method was proposed, in order to overcome some problems due to observability singularity set. This method is based on an immersion with a stable fictitious dynamics initialized at the equilibrium point. The inversion of the obtained diffeomorphism is not, generally, as simple as that in the Lorenz system with $x_{1}$ as output. In a future work, a numerical solution of the inverse diffeomorphism will be investigated by taking into account the particular structure of the obtained diffeomorphism.

Acknowledgements: The work has been funded by the Sectoral Operational Programme Human Resources Development 2007-2013 of the Ministry of European Funds through the Financial Agreement POSDRU/159/1.5/S/132395.

\section{References}

[1] G. Alvarez, F. Montoya, M. Romera, and G. Pastor, "Cryptanalysis of an ergodic chaotic cipher," Physics Letters A, vol. 311, pp. 172-179, 2003. 
[2] V. Andrieu and L. Praly, "Note on diffeomorphism extension for observer design," hal-00957648, 2014.

[3] J. Back and J. Soe, "Immersion of nonlinear systems into linear systems up to output injection: characteristic equation approach," International Journal of Control, vol. 77, no. 8, pp. 723-734, 2004.

[4] J. Back, K. Yu, and J. Soe, "Dynamic observer error linearization," Automatica, vol. 42, no. 12, pp. 2195-2200, 2006.

[5] M. Baptista, "Cryptography with chaos," Physics Letters A, vol. 240, pp. 50-54, 1998.

[6] V. Bijalwan, P. Kumari, J. Pascual, and V. B. Semwal, "Machine learning approach for text and document mining," arXiv preprint arXiv:1406.1580, 2014.

[7] M. Fliess, C. Join, and H. Sira-Ramirez, "Nonlinear estimation is easy," International Journal of Modelling Identification and Control, vol. 4, no. 1, pp. 12-27, 2008.

[8] L. Fridman, J. Moreno, and R. Iriarte, Sliding Modes after the first Decade of the 21st Century: State of the Art. Series: Lecture Notes in Control and Information Sciences, V 412, Springer, 2011.

[9] M. Frunzete, J.-P. Barbot, and C. Letellier, "Influence of the singular manifold of observable states in reconstructing chaotic attractors," Physical Review E, vol. 86, 2012.

[10] J.-P. Gauthier, H. Hammouri, and S. Othman, "A simple observer for nonlinear systems with applications to bioreactors," IEEE Transactions on Automatic Control, vol. 37, no. 6, pp. 875-880, 1992.

[11] R. Hermann and A. Krener, "Nonlinear controllability and observability," IEEE Transactions on Automatic Control, vol. 22, no. 5, pp. 728$740,1977$.

[12] P. Kumari and V. Abhishek, "Brainwave based user identification system: A pilot study in robotics environment," Robotics and Autonomous Systems, vol. 65, pp. 15-23, 2015. 
[13] C. Letellier and L. A. Aguirre, "Symbolic observability coefficients for univariate and multivariate analysis." Physical Review E, vol. 79, no. 066210, 2009.

[14] A. Levant, "Higher-order sliding modes, differentiation and outputfeedback control," International Journal on automatic, vol. 76, no. 9/10, pp. 924-941, 2003.

[15] — _ "Homogeneity approach to high-order sliding mode design," Automatica, vol. 41, pp. 823-830, 2005.

[16] A. Levant and M. Livne, "Exact differentiation of signals with unbounded higher derivatives," IEEE TAC, vol. 57, no. 4, pp. 1076-1080, 2012.

[17] M. L'Hernault, J.-P. Barbot, and A. Ouslimani, "Feasibility of analogue realization of a sliding mode observer: application to data transmission," IEEE Transaction on Circuit and System - I, vol. 55, no. 2, pp. 614-624, 2008.

[18] H. Nijmeijer and I. Mareels, "An observer looks at synchronization," IEEE Transactions on Circuits and Systems-1: Fundamental theory and Applications, vol. 44, no. 10, pp. 882-891, 1997.

[19] L. Pecora and T. Carroll, "Synchronization in chaotic systems," Physical Review Letters, vol. 64, no. 8, pp. 821-824, 1990.

[20] W. Perruquetti and J.-P. Barbot, Sliding Mode Control in Engineering. Marcel Dekker, 2002.

[21] A. Rapaport and M. Maloum, "Design of exponential observers for nonlinear systems by embedding," International Journal of Robust and Nonlinear Control, vol. 14, pp. 273-288, 2004.

[22] A. Rapaport and V. Maloum, "Embedding for exponential observers of nonlinear systems," Proceedings of the 39th IEEE CDC, Syndey, 2000.

[23] R. Tami, D. Boutat, and G. Zheng, "Extended output depending normal form," Automatica, 2013.

[24] A. Ticlea, "Extended output depending normal form," Ph D Thesis INPG 22 September 2006, 2006. 